Revue

de Sémantique

et Pragmatique
Revue de Sémantique et Pragmatique

39 | 2016

Varia

\title{
Rapports dialogiques entre narrateur et personnage dans un récit en Je : Quij'ose aimer d'Hervé Bazin
}

Dialogic relationships between narrator and character in a $1^{\text {st }}$ person narrative: Qui j'ose aimer by Hervé Bazin

\section{Ligia Stela Florea}

\section{OpenEdition}

Journals

Édition électronique

URL : http://journals.openedition.org/rsp/391

DOI : 10.4000/rsp.391

ISSN : $2610-4377$

Éditeur

Presses universitaires d'Orléans

Édition imprimée

Date de publication : 1 octobre 2016

Pagination : $19-38$

ISSN : 1285-4093

Référence électronique

Ligia Stela Florea, «Rapports dialogiques entre narrateur et personnage dans un récit en Je : Qui j'ose aimer d'Hervé Bazin », Revue de Sémantique et Pragmatique [En ligne], 39 | 2016, mis en ligne le 01

octobre 2017, consulté le 19 avril 2019. URL : http://journals.openedition.org/rsp/391 ; DOI : 10.4000/ rsp.391 


\title{
RAPPORTS DIALOGIQUES ENTRE NARRATEUR ET PERSONNAGE DANS UN RÉCIT EN JE : QUI J'OSE AIMER D'HERVÉ BAZIN
}

\author{
Ligia Stela Florea
}

Centre de Linguistique romane et Analyse du discours (CLRAD) Université Babeş-Bolyai, Cluj-Napoca (Ro)

\section{ENTRE ROMAN-TÉMOIGNAGE ET ROMAN POÉTIQUE}

Qui j'ose aimer illustre un genre de discours romanesque né à la moitié du siècle dernier avec le premier roman d'Hervé Bazin'1 . Ce genre, appelé « romantémoignage », ne retrace pas une histoire exemplaire aux résonances universelles mais l'expérience authentique d'un héros-narrateur « partial et passionnel » (Albérès 1968 : 282). La vision réaliste ne procède plus du « milieu » mais de l'individu, un héros en conflit avec le milieu, l'époque ou sa propre existence : Jean Rezeau de Vipère au poing, Arthur Gérane de La tête contre les murs, Constance Orglaise de Lève-toi et marche, Isabelle Duplon de Qui j'ose aimer.

L'investissement émotionnel du narrateur prête un certain lyrisme à cette prose romanesque ; en y ajoutant la dimension symbolique et la structure cir-

${ }^{1}$ Vipère au poing paraît en 1948 et obtient un succès immédiat. Les romans qui suivent (La tête contre les murs en 1949, La mort du petit cheval en 1950, Lève-toi et marche en 1952, L'huile sur le feu en 1954, Qui j'ose aimer en 1956) marquent, comme ceux de Georges Arnaud, Roger Peyrefitte ou Jean Hougron, l'avènement d'un nouveau réalisme. Il se distinguait tant du roman «tragique » (Bernanos, Mauriac) que du roman de la condition humaine (Malraux, Saint-Exupéry) et s'opposait radicalement au « roman-fresque », dérivé du naturalisme (Romains, Duhamel). 
culaire on obtient, selon Tadié (1997) le profil du « roman poétique ». À cette double filiation générique se joignent des propriétés stylistiques unanimement reconnues mais qui n'ont pas été explorées en profondeur : la verve du narrateur-témoin, qui s'associe chez Hervé Bazin à un admirable sens du détail ; l'esprit lucide et critique en perpétuel éveil qui prend tantôt le ton d'un humour enjoué, tantôt celui d'une ironie mordante tenant de la raillerie la plus cruelle. Sur quels mécanismes linguistiques reposent les caractéristiques qui font l'originalité de ce discours romanesque ? C'est la question à laquelle la présente étude se propose de répondre en interrogeant les rapports dialogiques qui se nouent entre narrateur et personnage, à l'aide des instruments fournis par la linguistique du texte et les théories de l'énonciation.

Qui j'ose aimer est un roman autodiégétique ou homodiégétique à narrateur protagoniste (Genette 1983) qui adopte la base temporelle de la narration classique PS/IMP. Néanmoins, il n'est pas toujours facile de distinguer les deux plans narratifs : le premier, qui accueille l'enchaînement temporel et causal des faits racontés, et le second plan où se mêlent descriptions et explications du narrateur, perceptions et réflexions de l'héroïne. Vu la forte implication subjective du narrateur intradiégétique, on assiste ici à une étroite intrication des points de vue du Je narrateur et du Je personnage. Il en résulte un « fondu enchaîné énonciatif » (Rabatel 2001), phénomène connexe de celui qu'on peut observer dans les récits hétérodiégétiques à focalisation interne, où narrateur et personnage sont des instances distinctes l'une de l'autre.

Nous allons essayer de démêler ce « fondu énonciatif » en mettant en œuvre les concepts théoriques et méthodologiques de la pragmatique énonciative. Notre approche du texte narratif repose principalement sur la théorie de «l'effet point de vue », élaborée par Alain Rabatel entre 1998 et 2008. Nous commençons donc par en rappeler les grandes lignes tout en insistant sur les aspects qui intéressent de près notre approche. Les réflexions autour de l'incipit du roman nous permettront ensuite de préfacer l'analyse de texte qui parcourt trois étapes et qui porte sur la construction des points de vue du Je narré et du Je narrant et sur les rapports dialogiques qui sous-tendent la dynamique textuelle.

\section{POINT DE VUE ET RAPPORTS NARRATEUR/PERSONNAGE}

Selon Rabatel (2008 : 33), « la problématique du point de vue est essentielle à la bonne interprétation des textes narratifs ». L'approche énonciative et pragmatique qu'il met en place dépasse les conceptions immanentistes du récit, déplaçant le centre d'intérêt sur les interactions que le narrateur noue avec ses personnages et sur les stratégies interprétatives visant à détecter les inférences construites par le texte. 
Les perceptions/pensées représentées ou point de vue (dorénavant pdv) sont, à côté du monologue intérieur et du discours indirect libre, des modalités de représentation de la subjectivité dans le texte narratif. Une perception représentée est une perception qui n'est pas simplement énoncée mais comporte une expansion où sont décrits divers aspects de la perception initiale et/ ou sont commentées certaines de ses caractéristiques. La construction textuelle d'une telle perception fait intervenir, selon Rabatel (1998:25), quatre grands facteurs linguistiques : (i) l'aspectualisation de l'objet focalisé, (ii) le marquage de l'opposition entre le premier plan de la narration et le second plan, (iii) la présence des formes temporelles-aspectuelles de visée sécante, notamment des formes d'imparfait, (iv) une relation d'anaphore associative entre perceptions représentées au second plan et perceptions assertées au premier plan.

Il convient de souligner deux caractéristiques de ce modèle : primo, il concernait au départ les récits hétérodiégétiques, c'est-à-dire les " récits à la $3^{\mathrm{e}}$ personne », et secundo, contrairement à l'approche traditionnelle du point de vue, qui visait la recherche du sujet percevant (qui voit) ou pensant (qui sait), l'approche de Rabatel vise avant tout la recherche du mode de référenciation de l'objet perçu.

Ce qui apparaît déterminant ce n'est plus dès lors « qui » voit ou « qui » sait, c'est l'analyse concrète de la référentialisation du focalisé, et, à partir d'elle, le repérage de l'énonciateur responsable des choix de référentialisation. (Rabatel $1998: 58-59$ )

La référenciation des objets de discours est fonction de « la position de l'énonciateur sur les objets de discours » et concerne, selon Rabatel (2013 : 140) : « les choix de catégorisation, de qualification, de modalité et de modalisation, d'ordre des mots et des prédications ou de mise en relief ». Dans les récits à la $3^{\mathrm{e}}$ personne, il y a un locuteur (L1) et deux types d'énonciateurs (E1, e2) : l'instance L1/E1 correspond au narrateur et l'instance e2 correspond au personnage qui n'est pas locuteur.

Dans un récit en je, il y a un locuteur-énonciateur qui se pose alternativement en narrateur (Je narrant) et personnage (Je narré). Si l'on peut y retrouver à peu près les mêmes modalités de représentation narrative que dans le roman à la $3^{\text {e }}$ personne - récit consonant (Cohn 1981), perceptions représentées (point de vue), monologue cité (discours direct), monologue narrativisé (discours indirect libre $)^{2}$ - la facture homodiégétique du roman rend assez problématique la distinction entre Je narrant et Je narré et, par conséquent, le repérage du pdv représenté du Je narré. Pour mieux cerner cette dualité, nous partons de la formule

${ }^{2}$ Ces modes de représentation narrative, Rabatel va les intégrer dans « une approche unifiante du point de vue » $(1999,2000)$ reposant sur la triade: point de vue raconté (récit consonant), point de vue asserté (discours rapporté et discours auctorial) et point de vue représenté (perceptions représentées et discours indirect libre). 
dont se sert Alain Rabatel (1998 : 10) pour définir la condition du narrateur Janus bifrons :

objectif lorsqu'il s'en tient au récit des faits, homologue à l'enchaînement des actions [...] et subjectif par le biais des choix narratifs, du mode de donation des référents, des évaluations et modalisations qui construisent un «discours [sur le] récit. »

Il est tentant de considérer que, dans les récits en je, ces deux facettes du narrateur se répartissent naturellement entre le Je narrant et le Je narré. En réalité les relations entre les deux instances sont beaucoup plus complexes, car elles puisent leur origine dans le dialogisme interne au locuteur-narrateur, tel qu'il a été défini par la théorie polyphonique de l'énonciation ${ }^{3}$.

Si dans son ouvrage de 1998, Rabatel ne s'occupe guère du syncrétisme entre Je narré et Je narrant, il se penche plus longuement sur ce phénomène dans une étude publiée en 2000 dans La lecture littéraire, pour y revenir dans le second volume de son Homo narrans au chapitre 7, intitulé « Bélligérances entre perspectives du narrateur et du personnage » :

Il [est] stratégiquement intéressant de s'arrêter sur l'intérêt des brouillages qui affectent le récit en je, puisque le travail de sape opéré à partir du syncrétisme des personnes, des rôles et des instances produit des interrogations majeures. (2008: 514)

En examinant quelques textes représentatifs, Rabatel constate que les brouillages qui affectent les récits homodiégétiques sont dus à ce que la frontière entre le mode et la voix, au sens de Genette (1972), tend à s'estomper. Tout en soulignant la nécessité de dissocier le Je narrant et le Je narré, Rabatel reconnaît que cette distinction, importante sur le plan théorique, s'avère problématique sur le plan de la praxis, vu que dans l'activité de narration « cette distinction n'est pas toujours nette » (2008: 517).

Nous voyons dans la dualité Je narrant/Je narré une relation dialogique entre une instance qui rapporte et une instance qui (re)vit les faits rapportés en expérimentateur, d'où l'alternance, à peine perceptible parfois, entre une modalité de type telling et une modalité de type showing. Telling c'est dire ce qui se passe (énoncés de faire) mais aussi à quel endroit et à quel moment cela se passe (énoncés d'état), alors que showing c'est montrer comment tout cela est vu, perçu, ressenti hic et nunc par le protagoniste. Contrairement au cas des récits à la $3^{\mathrm{e}}$ personne, où l'on peut déceler, selon Rabatel (2013), un dire et deux montrer, répartis sur deux instances : locuteur-énonciateur premier (narrateur)

${ }^{3}$ On pense d'abord à la distinction que fait Ducrot (1984) entre locuteur et énonciateur, le premier étant à l'origine de l'ancrage référentiel du discours et l'autre, à l'origine des modalisations et des qualifications. Mais on peut penser tout aussi bien à la distinction entre locuteur en tant que tel et locuteur en tant qu'être du monde. 
et énonciateur second (personnage non locuteur), dans le cas des récits à la 1ère personne, où les deux instances sont en syncrétisme, le dire est typiquement associé au locuteur-narrateur et le montrer, à l'énonciateur-personnage.

Pour distinguer ces deux instances nous allons prendre en compte : (i) le mode de référenciation des objets de discours, (ii) le degré d'implication subjective-affective du $J e$, qui entraîne une polarisation des marques (le DIL, les marqueurs argumentatifs, par exemple, sont spécifiques du Je narré), et (iii) les particularités stylistiques qui séparent une narration de facture plutôt classique de la confession pleine de verve, de vivacité et d'humour d'une adolescente pas tout à fait sortie de l'enfance.

\section{L'INCIPIT DE QUIJ'OSE AIMER}

Berthe n'apercevait rien, vous pensez bien : elle est myope, aussi. Les pieds prudemment posés à un mètre du bord, les mains sur le ventre et se triturant l'une l'autre, elle dodelinait de la tête, elle plissait les yeux, elle faisait de grands efforts pour sembler intéressée, en murmurant comme d'habitude :

« Tu crois, Isa? Tu crois? »

Je ne croyais rien. J'avais des yeux pour voir et je les voyais très bien tous les deux, là, au fond de l'Erdre, sous le treillage de la nasse : un long qui s'effilait, immobile, le nez sur les ardillons du goulot et un rond qui tournait frénétiquement, dans tous les sens, avec des miroitements mordorés ; compère Brochet et commère la Tanche, la seconde assez grosse pour ne pas m'être livrée dans le ventre du premier, mais, apparemment très effrayée du voisinage. Quant à la nasse, je la reconnaissais bien aussi, à son volume, à la forme de ses mailles : seul, M.Ténor en avait de ce modèle, et comme durant les vacances, chaque matin, vers onze heures, on le voyait godiller de place en place sur son sabot vert, il valait mieux faire vite si nous voulions lui économiser le beurre.

« Fait froid, Isa ? Fait froid ! » fit Berthe en me voyant porter la main au col de mon pull.

Il ne faisait pas chaud, certes. Les sauges tenaient ; l'iris jaune brûlait encore parmi les cannes à peine rouillées, à peine secouées par ces coups d'air qui prennent les roselières à rebrousse-poil. Mais le ciel avait un mois d'avance, noyait le soleil dans les gris fluides d'un automne précoce, à court de feuilles et d'oiseaux. Trop fraîche, cette eau, ni courante, ni dormante, qui ne sentait plus la vase et remontait, encore un peu crémeuse et repoussant doucement la canetille sur la berge ! Trop fraîche pour plonger. Mais comment faire autrement? Je n'avais pas de croc et d'ailleurs, la nasse était trop loin. Nous ne pouvions tout de même pas rater l'occasion de jouer un tour à l'ennemi et d'enrichir les menus de Nathalie, un peu trop portée sur les patates... Allons ! Le pull me jaillit des bras, ma jupe glissa, la combinaison suivit, aussitôt rejointe par ce soutien-gorge qui, du reste, n'avait jamais eu l'occasion de soutenir grand-chose depuis que, devenu trop petit pour maman, il avait repris du service en devenant trop grand pour moi. Frissonnante et les paumes sur les seins, j'hésitai avant d'enlever ma très blanche culotte. Mais la garder compliquait bien les choses; elle n'aurait pas le temps de sécher avant 
le déjeuner. Derrière nous, il n'y avait après tout qu'un jardin clos et, en face, de l'autre côté des chenaux, des îles, du bras canalisé, rien d'autre que le marais prolongé par l'immense prairie basse de la Glauquaie, déserte à l'infini, sans berger, sans vache et sans chien.

«Et tes cheveux! Tes cheveux ! protesta ma sœur, dans mon dos.

Tant pis ! La culotte venait de tomber ; mes chevilles, frottées l'une contre l'autre, se débarrassaient des souliers et, d'une vive détente, expédiaient dans la rivière, pour l'y rhabiller d'écume, ce corps qui ne m'inquiétait pas, mais dont l'eau indiscrète, durant une fraction de seconde me renvoya l'image, d'un rose sourd secrètement touché de sombre aux racines des membres.

(Qui j'ose aimer, chapitre I, pp.7-9)

La protagoniste de l'histoire, Isabelle Duplon a 18 ans et vit avec sa mère, sa sœur cadette Berthe et la bonne Nathalie à La Fouve, aux environs de Nantes. Après le divorce des parents, la vie des deux filles est bousculée à nouveau par le second mariage de la mère et l'apparition d'un beau-père qui va partager dorénavant leur vie et leur foyer. La drôle de pêche à laquelle se livre Isabelle sous le regard effrayé de sa petite sœur précède de près l'annonce du mariage, l'arrivée du couple et l'installation du beau-père dans la maison, perçue au départ comme une intrusion brutale dans cette société de femmes. Le beau-père, Maurice Méliset, est le fils de celui que la narratrice appelle M. Ténor et dont le domaine, La Glauquaie, avoisine celui des Duplon.

L'incipit in medias res, formule chère à Hervé Bazin, plonge d'emblée le lecteur dans l'univers du roman, repoussant l'exposition à un moment ultérieur (chapitre II); il s'ensuit que les informations concernant le cadre et le personnage-narrateur (âge, sexe, famille) nous seront livrées ici pêle-mêle à mesure qu'avance le récit.

Le lecteur se voit confronté d'emblée à un mélange de styles: d'un côté, un style « littéraire » très imagé et, de l'autre, un style familier teinté d'humour ou d'ironie. Dans ce roman-confession, où le narrateur se double d'une adolescente qui vit sa première expérience amoureuse, la tonalité oscille entre une gaieté enfantine encline au badinage et une révolte à peine contenue qui tourne à l'ironie et au sarcasme.

Le lexique est structuré autour de deux isotopies connexes : (i) la nature fluviale à l'automne et les activités afférentes (pêche et navigation), avec la présence des vocables techniques : nasse, treillage, ardillons, godiller, chenaux, bras canalisé... ; (ii) le corps féminin et les objets de vestimentation associés à l'acte de se dévêtir, avec la présence des verbes propres à vitaliser ces objets : jaillir, glisser, suivre, rejoindre, tomber, se débarrasser.

Bien que le roman adopte la base temporelle de la narration classique PS/IMP, il n'est pas très facile de distinguer les deux plans narratifs qui mêlent 
constamment voix du narrateur et points de vue du narrateur et du personnage. Pourtant, certains indices linguistiques et textuels laissent voir non seulement une certaine alternance mais une vraie interaction entre ces points de vue, interaction dont on va essayer de surprendre trois aspects : coénonciation ${ }^{4}$, domination du pdv du personnage et domination du pdv du narrateur.

\section{COÉNONCIATION ENTRE LES DEUX POINTS DE VUE}

Nous allons utiliser les critères déjà mentionnés (mode de référenciation du focalisé, mode de sémiotisation de la subjectivité, particularités stylistiques) pour identifier dans le texte ci-dessus les passages où le pdv du Je narré émerge du fondu énonciatif. L'analyse va porter essentiellement sur les séquences à l'imparfait (IMP) qui constituent l'arrière-plan de la narration; elles l'emportent en nombre sur les séquences au passé simple (PS) qui assurent la progression temporelle du récit.

4.1. Le premier paragraphe «Berthe n'apercevait rien, vous pensez bien... », qui brosse en quelques touches fugitives le portrait de la petite sœur d'Isabelle, construit le pdv du Je narrant, comme en témoigne l'adresse directe au lecteur, qui ne peut venir que du narrateur. La reprise - qui devient répétition - du pronom elle devant les verbes des trois dernières propositions juxtaposées traduit une insistance qui laisse deviner un mélange d'humour et de tendresse.

Tout le deuxième paragraphe du texte, à partir de Je ne croyais rien, est dominé en revanche par le pdv représenté du Je narré. L'émergence de son pdv est signalée par le changement de registre et par les éléments qui marquent une réaction du sujet d'énonciation au hic et nunc de la scène narrative, à savoir :

- la reprise diaphonique en DIL ( « je ne croyais rien ») qui fait écho au DD de Berthe ;

- la syntaxe suggérant la saisie sur le vif des perceptions ( « je les voyais très bien tous les deux, là, au fond de l'Erdre, sous le treillage de la nasse ») ;

- la personnification signalant que la perception est filtrée par le regard d'un enfant ( « compère Brochet et commère la Tanche, la seconde assez grosse pour ne pas m'être livrée dans le ventre du premier ») ;

${ }^{4}$ Ce terme est utilisé ici dans un sens légèrement différent de celui que lui prête la topique des postures énonciatives de Rabatel (2005). Coénonciation veut dire construction de deux points de vue qui se font équilibre et peuvent coexister dans les limites d'un seul énoncé. De même, plus loin, surénonciation veut dire construction d'un pdv dominant : le pdv du Je narré se pose en surplomb à l'égard du pdv du Je narrant. 
- la pointe d'ironie visant à ridiculiser le voisin d'en face (« M. Ténor, on le voyait godiller de place en place sur son sabot vert $\gg)^{5}$;

- le discours indirect libre sur un ton enjoué et familier (« il valait mieux faire vite si nous voulions lui économiser le beurre »).

4.2. Le troisième paragraphe, sans compter les brèves interpellations de Berthe, s'amorce toujours par le DIL du sujet expérimentateur, signalé par la modalisation : Il ne faisait pas chaud, certes. La phrase, centrée sur une négation descriptive, fait de nouveau écho au DD de Berthe : «Fait froid, Isa? Fait froid ! ».

En échange, la description du cadre, qui s'amorce par « Les sauges tenaient » et progresse avec intermittences jusqu'à « repoussant la canetille sur la berge », devrait plutôt être attribuée au Je narrant, vu que ce passage se remarque par un style plus recherché et surtout très imagé. Il fait voir d'autre part la distance psychologique que prend le sujet vis-à-vis des données de la référenciation : le Je narrant recompose le paysage par le biais du souvenir, de sorte qu'on a affaire à une description post hoc et non pas à une perception hic et nunc comme dans « Il ne faisait pas chaud, certes $»^{6}$.

Les métaphores qui colorent ce paysage d'automne, notamment la dernière, qui prête à la phrase des accents baudelairiens (« le ciel noyait le soleil dans les gris fluides... ») laissent transparaître une ombre de nostalgie : la nostalgie de l'été « trop court » qui, pour le Je narrant, serait la nostalgie d'un état de pureté et d'innocence.

Le pdv de l'héroïne refait surface avec la phrase nominale exclamative «Trop fraîche, cette eau... » et, en laissant de côté la relative, se déploie sur trois lignes jusqu'à la particule allons.

Trop fraîche, cette eau, ni courante, ni dormante, qui ne sentait plus la vase et remontait, encore un peu crémeuse et repoussant doucement la canetille sur la berge ! Trop fraîche pour plonger. Mais comment faire autrement ? Je n'avais pas de croc et d'ailleurs, la nasse était trop loin. Nous ne pouvions tout de même pas rater l'occasion de jouer un tour à l'ennemi et d'enrichir les menus de Nathalie, un

${ }^{5}$ Si godiller (manœuvrer une embarcation à l'aide d'une godille) est un verbe stylistiquement neutre, sabot est pris ici dans un sens (jouet d'enfant, toupie que l'on fait tourner en la fouettant) qui prête une valeur péjorative au syntagme son sabot vert.

${ }^{6} \mathrm{Si}$ le Je narrant ne prend plus le temps de rapporter les réactions du Je narré, comme au début du $2^{\mathrm{e}}$ paragraphe, il laisse transparaître en échange, par le biais de l'inverseur argumentatif mais, une vague appréhension : les couleurs de l'été sont encore vives (l'iris était jaune, les cannes à peine rouillées) mais l'automne aura vite fait de les «noyer » dans la grisaille. Un des motifs récurrents du roman, la grisaille, préfigure cette période indécise de la vie d'Isabelle où son hostilité envers Maurice Méliset commence à se teinter d'une certaine attraction physique (voir le début du troisième extrait, en 6). 
peu trop portée sur les patates... Allons !

Tout au début, on assiste à une étroite intrication entre pdv du personnage et pdv du narrateur. Si « Trop frâ̂che, cette eau... » et « Trop fraîche pour plonger » renvoient au discours intérieur du Je narré, la relative insérée entre les deux phrases nominales est une description attribuable au Je narrant. Le pdv représenté du Je narré se résout dans un monologue intérieur marqué par:

- mais + infinitif délibératif dans une interrogation tenant du discours autocentré, un DD ou un DIL (« Mais comment faire autrement ? ) ;

- trop loin, variable argumentative servant à appuyer la conclusion implicite de « je n'avais pas de croc et d'ailleurs la nasse était trop loin »);

- d'ailleurs, tout de même, connecteurs indiquant à leur tour un mouvement argumentatif (« Nous ne pouvions tout de même pas rater l'occasion... »);

- expression familière teintée d'humour (« jouer un tour à l'ennemi et enrichir les menus de Nathalie, un peu trop portée sur les patates ») ;

- allons, particule exclamative relevant de l'oral spontané.

4.3. Le passage suivant marque un net changement de perspective : des perceptions/ réflexions du Je narré on passe à la série d'actions qui forment le premier plan narratif : « Le pull me jaillit des bras, ma jupe glissa, la combinaison suivit, aussitôt rejointe par ce soutien-gorge... ». Le fait que tous ces PS ont pour sujet des objets de vestimentation, alors que la référence au possesseur revêt la forme d'un datif possessif (me jaillit) ou d'un article (ma jupe) semble traduire un souci d'objectivation propre au narrateur. Cette première série d'actions qui font avancer le récit s'achève sur un PS ayant pour sujet le Je narrant (j’hésitai), pour céder aussitôt la place à une délibération intérieure qui renvoie au pdv du Je narré :

Mais la garder compliquait bien les choses ; elle n'aurait pas le temps de sécher avant le déjeuner. Derrière nous, il n'y avait après tout qu'un jardin clos et, en face, de l'autre côté des chenaux, des îles, du bras canalisé, rien d'autre que le marais prolongé par l'immense prairie basse de la Glauquaie, déserte à l'infini, sans berger, sans vache et sans chien.

Les « embrayeurs » de ce pdv sont les connecteurs argumentatifs mais, après tout qui, en lien avec la négation restrictive et le prospectif (n'aurait pas le temps) servent à appuyer le constat qui amorce ce passage. Les marqueurs spatiaux derrière nous, en face connotent de leur côté la saisie sur le vif des perceptions et des pensées.

Le dernier paragraphe du texte mêle intimement les deux pdv, ce dont témoigne, au niveau du lexique, la coprésence de termes relevant du registre familier et littéraire. Ce qui confère sa note originale à cette prose narrative 
est justement le mélange de registres et la dimension poétique reposant sur la densité figurale :

Tant pis ! La culotte venait de tomber ; mes chevilles, frottées l'une contre l'autre, se débarrassaient des souliers et, d'une vive détente, expédiaient dans la rivière, pour l'y rhabiller d'écume, ce corps qui ne m'inquiétait pas, mais dont l'eau indiscrète, durant une fraction de seconde me renvoya l'image, d'un rose sourd secrètement touché de sombre aux racines des membres.

Le bref énoncé exclamatif tant pis fait écho aux avertissements de Berthe : comme dans les deux cas précédents, le discours direct du Je narré se convertit en discours intérieur de type DIL.

Bien que dominé par l'imparfait, ce passage observe l'opposition des plans narratifs :

- le premier plan, qui prolonge la succession chronologique des actions par le biais de trois IMP pittoresques et d'un PS. Les verbes tomber, se débarrasser, expédier, renvoyer se voient attribuer des sujets non animés (la culotte, mes chevilles, l'eau);

- le second plan, qui marque un « arrêt sur image » par le biais d'un imparfait descriptif (ce corps qui ne m'inquiétait pas) et d'un ample groupe nominal suggérant une perception visuelle du Je narré : « un rose sourd secrètement touché de sombre... ».

À part le début et la fin, le dernier paragraphe construit le pdv du Je narrant. Le syntagme démonstratif ce corps connote l'attitude détachée du narrateur, alors que les qualifications indiscrète et secrètement semblent renvoyer plutôt au pdv du personnage. L'eau est « indiscrète » pour une adolescente qui commence justement à découvrir son corps et qui est « secrètement » fascinée par les signes de sa féminité. Ainsi, mais, connecteur argumentatif et embrayeur du pdv, marque une opposition sur le plan énonciatif entre la relative en qui et la relative en dont : il signale que les deux pdv qu'il relie sont anti-orientés du point de vue argumentatif. Autrement dit, le Je narrant et le Je narré ont une aperception différente du corps de l'héroïne que la nature aquatique se charge de mettre en valeur ${ }^{7}$.

Au fond, ces variations sur le motif du corps - la partie de pêche servant de prétexte à la mise en exergue du corps d'Isabelle - ne font que préfigurer sa première expérience amoureuse, où elle va «plonger » la tête la première comme

${ }^{7}$ La conjonction, dans l'incipit de Qui j'ose aimer, d'un espace particulier (le bord de l'eau) et d'un moment privilégié (état de pureté et d'innocence) engendre l'une des significations symboliques du roman. Cette mise en valeur de la beauté féminine par la nature aquatique va tout à fait dans le sens de la conception de Gaston Bachelard (1993), qui associe la rivière à la nudité et à l'innocence de la femme (cf. Rus 2011). 
dans cette scène et, toujours comme ici, pour voler, car son amant sera nul autre que son beau-père, le second mari de sa mère. De ce point de vue, l'incipit de Qui j'ose aimer, comme celui de Vipère au poing ou de La tête contre les murs, construit ce qu'on pourrait appeler une scène emblématique.

\section{QUAND LE PDV DU JE NARRÉ PREND LE DESSUS}

5.1. Ce sont les scènes qui favorisent en général le dédoublement narrateur/ personnage ou, si l'on veut, l'émergence du pdv du Je narré, qui peut l'emporter parfois sur le pdv du Je narrant. Si dans l'incipit du roman les deux pdv se font équilibre dans une coénonciation sui generis, au IIIe chapitre, dans la scène où Isabelle, cachée derrière son cormier, contemple l'arrivée en barque des nouveaux-mariés, le pdv du personnage se pose en surplomb à l'égard du pdv du narrateur. Nous transcrivons le début de ce chapitre, puis l'essentiel de la scène en question :

Vers onze heures, l'inquiétude, puis les cris, puis les courts envols des effarvattes, reculant devant l'intrus de roseau en roseau, dénoncèrent son approche. Presque aussitôt je reconnus les bruits familiers : ce léger clapotis qui gonfle l'herbe [...], ce goutte à goutte des rames qui replongent, ce froissement d'eau déchirée par l'avance d'un bateau [...]. Enfin deux ombres chinoises glissèrent à fleur d'oseraie entre les grimaçants tétards rasés de frais par les vanniers.

Deux, dis-je. J'avais eu raison [...]. Mon instinct ne m'avait pas trompée qui [...] m'avait expédiée à la motte du cormier, d'où l'on peut dominer le marais et voir, à coup sûr, ce qui se passe du côté de La Glauquaie. De la Bernerie, « ils » avaient certainement filé sur Carquefou. Puisqu'ils nous avaient en somme annoncé leur mariage, à nous, les filles, bien obligées de les subir, il était facile de deviner qu'ils se trouvaient obligés d'en faire poliment part au seigneur d'en face, plus difficile à manier [...]. Et Maman, escortée par son chevalier, rentrait par la rivière, une fois de plus obligée de me trahir.

Nul doute : ils ont certainement eu du mal à convaincre le vieux [...]. Peut-être même leur a-t-il opposé un refus catégorique. Espoir suprême ! Malgré la publication des bans [...], je m'y raccroche tandis que la barque, maintenant en pleine rivière, s'avance sur un ciel renversé, très bleu, festonné de gros cumulus. Maman est assise à l'arrière ; Maurice Méliset rame en me tournant le dos ; mais ces bouffées d'air [...] qui portent au loin le moindre murmure, ne laissent monter aucun mot. Ce silence est de bon augure, avoue-t-il une défaite ? Ou au contraire, à force de se regarder, n'éprouvent-ils plus aucun besoin de parler ? La haine m'étouffe et j'invoquerais dieu ou diable pour obtenir un miracle qui fasse basculer dans le bouillon ce gandin, avec sa raie, son fil-à-fil et son col blanc, qui me permette d'aller l'empoigner par la cravate, de le ramener, tout boueux, tout honteux, sur la berge.

Mais, tandis que je m'efface instinctivement derrière le cormier pour ne pas être vue, la barque pique droit, sans même balancer, file vivement sur son erre. Il ne 
me sera même pas donné de la voir buter un peu sec ! L'animal [...] dérame à la perfection, accoste comme sur du feutre ; il est déjà à terre, il tire sur la chaîne et Maman s'avance sur la pointe des pieds [...].

«Ta main » gémit-elle.

Et le tutoiement me rallume. On la lui tend, la main ! On lui tend même les deux - en assurant la chaîne avec le pied. Elle saute, exagérant à plaisir une maladresse exquise [...]. Elle est... comment dire ? Elle est en ce moment la beauté même. Elle se détache sur ce fond d'eau comme l'Egyptienne de la fable sortie, vivante, de son miroir [...].

Et Maurice se relève, les mains en avant. Et les voilà devant moi, enlacés, entortillés l'un dans l'autre, tête dessus, tête dessous, pour un bouche-à-bouche écrasé, un gros plan de fin de mélo. Et je me surprends à compter les secondes, une, deux, trois, quatre, en les dévorant des yeux...

(Qui j'ose aimer, chap. III, pp. 25-30)

Ce qui frappe dès le prime abord c'est le changement de base temporelle : si le début du chapitre repose toujours sur la base PS/IMP, les moments forts de la scène narrative sont relatés au présent. L'hypotypose, soutenue aussi par les déictiques adverbiaux, crée l'impression d'une coïncidence parfaite entre les faits vécus et l'acte de narration.

Dans les deux premiers paragraphes, les rapports entre Je narré et Je narrant sont en gros ceux de l'incipit : le narrateur fixe les coordonnées temporelles et spatiales de la scène et prend en charge l'enchaînement temporel et causal des faits racontés. Quant à la description des signes qui annoncent l'arrivée du couple, elle relève, par les traits stylistiques, du Je narrant, mais, par la frâ̂cheur et la vivacité des images auditives (ce léger clapotis, ce goutte à goutte des rames, ce froissement d'eau...), elle tient du Je narré. On doit lui attribuer également le marqueur enfin, qui montre l'état d'inquiétude dit, énoncé dès la première ligne par le Je narrant.

Il convient de s'arrêter un peu sur les actes de réflexion qui font émerger le « monologue » du Je narré : l'acte du second paragraphe est un raisonnement, dont les étapes sont marquées par certainement, puisque, en somme, il était facile de deviner et qui reconstruit le trajet suivi par le couple avant de rentrer à La Fouve. L'acte du troisième paragraphe suppute les chances de réussite du projet de mariage, passant de l'hypothèse probable («Nul doute : ils ont certainement eu du mal à convaincre le vieux...») à l'hypothèse possible («Peut-être même leur a-t-il opposé un refus catégorique »). Entre les deux actes on constate des différences d'ordre sémantique et énonciatif. Si le premier est une déduction logique, le second est une conjecture. Les éléments cotextuels, y compris les temps verbaux, indiquent que le Je narré engage à chaque fois une autre relation avec le Je narrant: coénonciation dans un cas, surénonciation, dans l'autre. 
5.2. En effet, à partir du $3^{\mathrm{e}}$ paragraphe, le pdv du personnage l'emporte à tel point sur celui du narrateur qu'il détermine un changement de régime narratif : du récit rétrospectif on passe à un récit témoignage « en prise directe » sur les faits racontés. Les éléments qui assurent la coïncidence entre le monde raconté et l'acte de narration sont :

- l'hypotypose confortée par les déictiques adverbiaux (maintenant, en ce moment) ;

- les déictiques spatiaux (les voilà devant moi) et les syntagmes démonstratifs dénotant la proximité immédiate : ces bouffées d'air, ce silence, ce gandin, ce fond d'eau;

- les verbes évoquant l'approche de la barque et les gestes des deux personnages; ils sont ponctués d'expressions évaluatives qui soulignent la qualité des mouvements, déplaçant l'accent du telling (récit) au showing (description d'actions) :

- La barque, maintenant en pleine rivière, s'avance sur un ciel renversé, très bleu ;

- La barque pique droit, sans même balancer, file vivement sur son erre ;

- L'animal dérame à la perfection, accoste comme sur du feutre ;

- Maman s'avance sur la pointe des pieds, elle saute, exagérant à plaisir une maladresse exquise, elle se détache sur ce fond d'eau comme l'Egyptienne de la fable...

N'oublions pas cependant que les deux modalités sont inséparables, comme les deux instances du reste : si la façon de voir et de percevoir est celle du personnage, le contenu propositionnel de ces énoncés est bien le fait du narrateur.

5.3. Mais ce qui consacre la domination du showing c'est, comme dans l'incipit, la mise en scène des actes psychiques de la protagoniste.

Les actes de réflexion se traduisent par des interrogations déliberatives en rapport disjonctif et oppositif : "Ce silence est de bon augure, avoue-t-il une défaite ? Ou au contraire, à force de se regarder, n'éprouvent-ils plus aucun besoin de parler?». Puisque pour Isabelle 'défaite' signifie 'échec du projet de mariage', ce qu'elle désire et espère ardemment, l'axiologique négatif défaite devient synonyme de l'axiologique positif de bon augure.

Les réactions affectives (l'espoir de voir échouer ce mariage, la haine et la jalousie à l'égard de Maurice, l'amour et l'admiration à l'égard de la mère) se traduisent par :

- des énoncés exclamatifs (espoir suprême! ; il ne me sera même pas donné de la voir buter..! ; on la lui tend, la main!) ; 
- des appellatifs dévalorisants ce gandin, l'animal, qui réfèrent à Maurice, et le pronom ils entre guillemets du second paragraphe, qui réfère aux nouveaux-mariés. Les guillemets connotent à la fois la modalité axiologique et le discours rapporté : "ils » concentre les sentiments des femmes de La Fouve envers ceux qui menacent l'équilibre de leur foyer ;

- un style oralisant : inachèvement, incise métadiscursive et expression du haut degré («Elle est...comment dire ? Elle est en ce moment la beauté même ») ;

- la pointe d'humour revêtant une tonalité amère, presque sarcastique («Et les voilà devant moi, enlacés, entortillés l'un dans l'autre, tête dessus, tête dessous, pour un bouche-à-bouche écrasé... »).

Puisque la modalité du showing, associée au Je narré, et la modalité du telling, associée au Je narrant, sont en syncrétisme, les sentiments et les ressentiments du personnage-témoin où baignent tous les détails de la scène narrative s'expriment tantôt par le biais du showing, comme on l'a déjà vu, tantôt par le biais du telling ${ }^{8}$ :

- La haine m'étouffe et j'invoquerais dieu ou diable pour obtenir un miracle qui fasse basculer dans le bouillon ce gandin [...], aller l'empoigner par la cravate, le ramener tout boueux sur la berge ;

- Et le tutoiement me rallume;

- Et je me surprends à compter les secondes, une, deux, trois, en les dévorant des yeux...

Intrication du dire et du montrer, des pdv du narrateur et du personnage et de leurs marques stylistiques : le registre châtié se mêle au registre familier, perceptible tant au niveau de la syntaxe que du lexique (le vieux, l'animal, le bouillon, me rallume).

\section{QUAND LE PDV DU JE NARRANT PREND LE DESSSUS}

On peut rencontrer aussi le phénomène inverse : le pdv du Je narrant s'impose à tel point qu'il supplante complètement le pdv du Je narré. C'est le cas de la première séquence (division) du chapitre XIV, l'un des plus longs du roman. C'est aussi un chapitre clé, car il évoque les faits dont l'accumulation graduelle déclenche l'événement qui va bouleverser les rapports au sein de la famille et marquer un tournant dans la vie de l'hérö̈ne.

La première séquence met en place le discours du narrateur, qui inclut un récit itératif au présent, encadré par deux commentaires sur les événements à

${ }^{8}$ Pour les modes de sémiotisation du showing (montrer) et du telling (dire) nous renvoyons le lecteur à l'article de J.Jacquin et R.Micheli (2013) : «Dire et montrer qui on est et ce que l'on ressent ». 
venir. Le récit condense une période de trois mois de la vie des Duplon-Méliset, période située sous le signe de l'ambiguïté'. En revanche, la deuxième et la troisième division du texte nous font assister à de nouvelles scènes qui ont le rôle d'illustrer les dires du narrateur par deux moments que l'hérö̈ne va revivre hic et nunc. Ils sont annoncés par la séquence métadiscursive qui clôt le discours du narrateur : « ces grandes scènes [...] qui sont nos images d'Epinal ».

Et voici que pour la seconde fois j'éprouve de la confusion. J'aime me croire entière et logique ; je déteste me souvenir de ces eaux troubles, de ces remous qui ont empêché un moment ma vie de couler tout droit. Je sais bien qu'il me reste contre eux un recours : celui de l'anguille qui s'envase sous la crue et sait attendre la fin de l'hiver pour sortir des fonds. Mais comment oublier celui-là ?

Décembre, janvier, février [...]. Le temps hésite entre la pluie, la neige et le vent. Maman hésite entre l'un peu mieux et l'un peu moins bien, Maurice entre le tu et le vous, Nathalie entre la hargne et la résignation. Et du pavé luisant de Nantes aux glaises détrempées de La Fouve, de la salle au bureau, de la chambre bleue au cours Pigier [...], je vais, je viens, indécise et ne sachant pourquoi je suis si satisfaite d'être mécontente de moi.

De moi et, bien entendu, de tout le monde. De ma sœur vraiment trop lente à comprendre qu'il ne faut plus dire «Monsieur bis » [...]. De Nathalie vraiment sans charité, pressante, crochetant sans cesse vers le reproche, l'aigre allusion à ma défection [...]. De Maurice vraiment trop différent de lui-même, ni beau-père, ni patron, ni ami, un peu tout à la suite, un peu tout à la fois, sans compter les fugitives apparitions de ce quatrième personnage trop gentil pour n'être pas galant [...] et souriant du même sourire devant ma mère ou devant moi.

Du même sourire. Ce qui s'allume vacille souvent dans la lueur de ce qui s'éteint. On commence avant même d'achever d'en finir et la vie continue, qui substitue sans cesse et ne fait pas de cassure. Comment savoir ? Comment prévoir ? Et de quoi même se souvenir qui mérite d'être avoué ? Ce qui nous explique, ce ne sont pas tellement ces grandes scènes qu'instinctivement la mémoire - cette rouée - a choisies et qui sont, à notre propre usage, nos images d'Epinal. Mais il faut bien des repères et j'en vois quelques-uns...

Cette salle de tribunal correctionnel, d'abord. Le président interroge l'accusée [...]. Maurice [...] bâille, se cure un ongle à l'aide d'un autre ongle, donne des coups de menton pour saluer à la ronde quelques confrères. C'est la première fois que je le vois en robe, officiant - sans gloire - à son banc.

Mais il se lève, calme, avare de ses mains qui restent plongées dans ses manches [...] ; il parle et le prétoire se remplit de sa voix, moins sonore qu'efficace, laçant

${ }^{9}$ Est suggestif en ce sens, dans le second paragraphe, le parallélisme qui sous-tend deux séries de structures : (1) SN hésite entre SN et SN ; (2) du SN au SN je vais, je viens. À ces régularités assurées par la syntaxe poétique s'ajoute un effet de contraste produit par le lexique : « indécise et ne sachant pourquoi je suis si satisfaite d'être mécontente de moi ». 
la phrase, la nouant sur l'argument [...]. C'est encore un autre homme, contre qui je n'ai rien.

Puis voici la chambre bleue. Je rentre de Nantes, j'ai devancé Maurice qui gare sa voiture [...]. Nat se détourne à peine [...]. Berthe réclame des bonbons que je lui ai promis. Maman, qui serait plutôt dans un bon jour, abandonne un de ces affreux petits romans qu'elle affectionne, m'accable de menues questions. Qu'avons-nous mangé à midi ? Suis-je contente de mon travail ? Combien de visites dans la journée ? [...]. Maman change vite de sujet mais non d'inquiétude :

«Vous recevez aussi des clientes?»

(Qui j'ose aimer, chap. XIV, pp. 120-123)

La première division du texte met en place le point de vue asserté du narrateur qui relève pour l'essentiel du telling. Ce qui tient du showing ce sont les commentaires sur le récit en train de se faire : ils laissent transparaître l'état d'esprit du narrateur par le biais d'un mouvement délibératif tourné autant sur le récit que sur lui-même.

Le mouvement délibératif s'amorce à la fin du premier paragraphe («Mais comment oublier celui-là ? ») et se poursuit dans le quatrième paragraphe par trois propositions infinitives qui complètent le discours autocentré du Je narrant : «Comment savoir? Comment prévoir? Et de quoi même se souvenir qui mérite d'être avoué ?».

La modalité du telling prend le dessus dans les passages qui relèvent du registre figural et sentencieux. Le registre figural se construit autour d'une métaphore filée ( « ces eaux troubles, ces remous...; l'anguille qui s'envase sous la crue... ») et couvre une bonne partie du premier paragraphe, alors que le registre sentencieux occupe tout le quatrième paragraphe : « Ce qui s'allume vacille souvent dans la lueur de ce qui s'éteint... ; « Ce qui nous explique, ce ne sont pas tellement ces grandes scènes qu'instinctivement la mémoire a choisies... ».

Relève également du telling la manière dont le narrateur fait part au lecteur des sentiments qu'il éprouve en évoquant les faits relatés: « Et voici que pour la seconde fois j'éprouve de la confusion. J'aime me croire entière et logique ; je déteste me souvenir de ces eaux troubles... ». Ce sont là des sentiments assertés auxquels succède, à la fin du quatrième paragraphe, un sentiment montré par le biais d'un énoncé nominal exclamatif : « la mémoire - cette rouée ».

La fin du quatrième paragraphe renforce le caractère métadiscursif de l'intervention auctoriale: d'abord, le qualificatif que la narrateur attribue à la mémoire est une allusion assez transparente au caractère subjectif des choix narratifs. Ensuite, la référence explicite à l'objet préférentiel de ces choix, les scènes, vise - malgré le tour restrictif - à en souligner la fonction explicative 
dans le roman. Les scènes sont des repères qui orientent le lecteur, l'aidant à déchiffrer les significations construites par le texte.

Quant au récit itératif, où les fluctuations qui affectent l'état ou les attitudes des personnages semblent suivre les montées et les descentes de l'Erdre, il est peu favorable à l'émergence du showing, compris - de manière générale - comme un mouvement expressif tourné vers le moi du narrateur ou du personnage. On a bien affaire ici à certains choix narratifs, mais ces choix, tout en étant liés au point de vue du narrateur, n'en relèvent pas moins du telling, car ils font l'objet d'un acte d'assertion.

Enfin, ce troisième extrait nous fait assister à une dissociation nette entre Je narrant et Je narré. Des indices aussi bien énonciatifs que graphiques séparent la séquence qui construit le point de vue asserté du narrateur des deux scènes où émerge le point de vue représenté du personnage. Les marqueurs de ce pdv sont en gros ceux que nous avons relevés dans le texte du deuxième extrait : l'hypotypose soutenue par la deixis temporelle et spatiale, d'une part, les syntagmes démonstratifs, l'anaphore associative et les présentatifs, d'autre part.

La dernière division du texte apporte cependant un élément nouveau par rapport à l'extrait précédent : elle ne met pas en scène le discours intérieur de la protagoniste mais les paroles d'un autre personnage. Condamnée à l'immobilité par une maladie incurable, la mère assaille sa fille de questions qui dissimulent à peine une certaine inquiétude. Représentées au début sur le mode du discours direct libre, les questions de la mère suivent un crescendo qui atteint le point culminant par le DD « vous recevez aussi des clientes ? » qui révèle la cause de son inquiétude ${ }^{10}$.

\section{CONCLUSION}

Mettre à jour les rapports dialogiques que le narrateur noue avec ses personnages, c'est l'une des conditions essentielles pour la bonne interprétation du texte narratif. Cette tâche devient plus difficile sinon problématique dans le cas des récits homodiégétiques, où le Je narrateur et le Je personnage fonctionnent en syncrétisme.

Les trois analyses de texte, qui mettent en œuvre les acquis de la pragmatique énonciative, montrent qu'il est possible non seulement de dissocier les deux instances mais aussi que les mécanismes linguistiques et textuels qui sous-tendent leurs rapports sont en gros analogues à ceux que l'on peut observer dans les récits à la $3^{\mathrm{e}}$ personne (cf. Florea 2005 et 2007). Par ses propriétés

${ }^{10}$ Nous tenons à remercier notre relecteur pour la patience et l'intérêt avec lesquels il s'est penché sur cette contribution, et pour les remarques pertinentes dont elle a tiré un réel profit. 
structurales et stylistiques, le roman Qui j'ose aimer d'Hervé Bazin offre le support idéal pour une telle approche et les opérations effectuées en vue d'une plus profonde exploration du texte permettent de mieux cerner les mécanismes linguistiques et textuels qui sous-tendent ces propriétés.

Le fait d'identifier le genre (espèce en l'occurrence) constitue un point de départ nécessaire, car il explique dans une grande mesure la coexistence dans Qui j'ose aimer des registres littéraire et familier : si le roman-témoignage privilégie le style oralisant et familier, le roman poétique s'oriente de préférence vers un style classique riche en images. Mais l'essentiel pour nous c'était de définir la dualité Je narrant/Je narré en la plaçant successivement sous le signe de plusieurs relations : objectivité et subjectivité, locuteur et énonciateur, telling et showing.

Notre hypothèse de départ est que, dans un récit homodiégétique, il y a un $J e$ qui rapporte ce qui se passe, en articulant énoncés de faire et énoncés d'état, et un $J e$ qui voit, perçoit, ressent les choses rapportées ou décrites en expérimentateur. Pour distinguer les deux instances on a mis à profit les concepts méthodologiques de «l'effet point de vue » (décrochage énonciatif entre premier et second plan, mode de référenciation du focalisé, point de vue représenté, point de vue asserté, effets argumentatifs indirects) et les pratiques des approches centrées sur le mode de sémiotisation de l'identité et de l'émotion.

$\mathrm{Vu}$ le syncrétisme entre Je narrant et Je narré, l'intrication de leurs points de vue est, naturellement, plus étroite et plus complexe que dans les cas où narrateur et personnage sont des instances distinctes. On peut déceler cependant dans ce « fondu enchaîné énonciatif » (Rabatel 2001) l'émergence d'un pdv du Je narré en rapport de coénonciation ( ${ }^{\text {er }}$ extrait) ou de surénonciation $\left(2^{\mathrm{e}}\right.$ extrait) avec le pdv du Je narrant. Mais l'analyse du $3^{\mathrm{e}}$ extrait montre qu' on peut assister même à une dissociation radicale de ces points de vue, ce qui projette une nouvelle lumière sur les rapports dialogiques narrateur/ personnage dans les récits en $J e$. 


\section{RÉFÉRENCES BIBLIOGRAPHIQUES}

Albérès, R.-M. (1968), Istoria romanului modern, Bucureşti : Editura pentru Literatură Universală.

Bachelard, G. (1993), L'eau et les rêves. Essai sur l'imagination de la matière, Paris: Librairie José Corti.

Bazin, H. (1956), Qui j’ose aimer, Paris : Editions Bernard Grasset.

Cohn, D. (1981), La transparence intérieure. Modes de représentation de la vie psychique dans le roman, Paris : Editions du Seuil.

De Chanay, H., Colas-Blaise, M., Le Guern, O. (dir.) (2013), Dire / Montrer - Au cœur du sens, Chambéry : Université de Savoie.

Ducrot, O. (1984), Le dire et le dit, Paris : Editions de Minuit.

Florea, L.-S. (2005) « Narration au présent, deixis fictionnelle et point de vue », in Revue de sémantique et pragmatique, 17 : 69-88.

I d e m (2007), « Jeu des points de vue et mise en intrigue dans L'homme de Londres de Georges Simenon, in Studia Universitatis Babeş-Bolyai - seria Philologia, 1:193-209.

Genette, G. (1972), Figures III, Paris : Editions du Seuil.

I d e m (1983), Nouveau discours sur le récit, Paris : Editions du Seuil.

Jacquin, J. et Micheli, R. (2013), « Dire et montrer qui on est et ce que l'on ressent : une étude des modes de sémiotisation de l'identité et de l'émotion », in Hugues de Chanay, Marion Colas-Blaise, Odile le Guern (dir.), Dire/Montrer - Au cœur du sens : 67-92.

Rabatel, A. (1998), La construction textuelle du point de vue, Lausanne-Paris : Editions Delachaux-Niestlé.

I d e m (1999), Mais dans les énoncés narratifs : un embrayeur du point de vue et un organisateur textuel », Le Français moderne, LXVII, 1 : 49-60.

I d e m (2000), « Un deux, trois points de vue ? Pour une approche unifiante des points de vue narratifs et discursifs », La lecture littéraire, 4 : 195-254.

I d e m (2001), « Fondus enchaînés énonciatifs. Scénographie énonciative et point de vue », Poétique, $126: 151-173$.

I d e m (2005), « Analyse énonciative et interactionnelle de la confidence. À partir de Maupassant », Poétique, 141 : 93-113.

I d e m (2008), Homo narrans. Pour une analyse énonciative et interactionnelle du récit, Tome II Dialogisme et polyphonie dans le récit, Limoges : Editions Lambert-Lucas. 
I d e m (2013), « Les relations dire/montrer au prisme de l'analyse énonciative des points de vue perceptuels », in Hugues de Chanay, Marion Colas-Blaise, Odile le Guern (dir.), Dire/Montrer - Au cœur du sens : 137-158.

Rus, A. (2011), Poétique de l'espace chez Hervé Bazin et Jean Rouaud, Cluj-Napoca : Casa Cărţii de Stiinţă.

Tadié, J.-Y. (1997), Le roman au XX' siècle, Paris : Pierre Belfond.

I d e m (2005), Le récit poétique, Paris : Gallimard. 